Referencia para citar este artículo: Dukuen, J. (2018). Socialización política juvenil en un colegio de clases altas (Buenos Aires, Argentina). Revista Latinoamericana de Ciencias Sociales, Niñez y Juventud, 16(2), 867-880. doi:https://doi.org/10.11600/1692715x.16215

\title{
Socialización política juvenil en un colegio de clases altas (Buenos Aires, Argentina)*
}

\author{
JUAN DUKUEN *** \\ Investigador del Consejo Nacional de Investigaciones Científicas y Técnicas (Conicet), Argentina.
}

\begin{abstract}
Artículo recibido en febrero 23 de 2018; artículo aceptado en abril 6 de 2018 (Eds.)
\end{abstract}
- Resumen (analítico): en este artículo se presentan hallazgos de una investigación sobre socialización política estudiantil en un colegio privado de clases altas de la Ciudad de Buenos Aires $y$ de tradición británica, en referencia a una práctica propia de gobierno escolar: las elecciones para capitanes de colegio. A partir de un trabajo de campo realizado entre 2016 y 2017 que comprendió entrevistas, observación participante y análisis de documentos, se analizan las cartas de postulación de candidatos a capitanes, para dilucidar los sentidos que le otorgan a esta modalidad de representación escolar. Se destaca que el liderazgo, la capacidad de comunicación y la antigüedad, constituyen sentidos atribuidos a las capitanías. Se concluye relacionándolos con procesos más amplios de socialización política juvenil en clases altas, que conducen a líneas futuras de investigación.

Palabras clave: joven, política, escuela, clase alta, liderazgo, socialización política, disposiciones políticas (Tesauro de Ciencias Sociales de la Unesco).

\section{Youth political socialization in a school for upper classes (Buenos Aires-Argentina)}

Abstract (analytical): This article presents findings of a research on youth political socialization in a private school of British tradition for upper classes in the City of Buenos Aires, about a specific practice of school government: elections for school captains. From a fieldwork carried out between 2016 and 2017 that included interviews, participant observation and document analysis, this paper analyzes the letters of nomination of candidates to captains in order to elucidate the meaning that students give to this practice of school representation. Highlight that leadership, communication skills and antiquity, constitute the senses attributed to the captaincies. It is concluded by relating them to broader processes of youth political socialization in the upper classes, which lead to future lines of research.

Key words: Youth, polítics, school, upper class, leadership, political socialization, political dispositions (Unesco Social Science Thesaurus)

\section{Socialização política juvenil em uma escola das classes superiores (Buenos Aires - Argentina)}

Resumo (analítico): este artigo apresenta os resultados de uma pesquisa sobre socialização política juvenil em uma escola privada das classes superiores da Cidade de Buenos Aires e da

Este artículo basado en un reporte de caso se fundamenta en una investigación realizada por el autor titulada «Moral, escuela y política en jóvenes de clases altas. Un estudio sobre la incorporación de esquemas morales y disposiciones políticas en una escuela secundaria de Ciudad de Buenos Aires» y forma parte de un estudio de mayor escala Pict-ANPCYT-Argentina No 2012-2751 dirigido por Dra. Miriam Kriger. Cuenta además con el aval del proyecto UE 22920160100005CO-CIS Conicet/Ides-Argentina dirigido por el Dr. S. Visacovsky. Fechas de la investigación: 1 marzo de 2016-15 de diciembre de 2017. Área de conocimiento: Sociología; subárea: Antropología.

** Dr. en Ciencias Sociales (Universidad de Buenos Aires), Investigador asistente del Consejo Nacional de Investigaciones Científicas y Técnicas (Conicet-Argentina) con sede en el CIS/Ides, Profesor Investigador de la Universidad de Buenos Aires. Orcid: 0000-0001-5089-1858. Índice H5: 6. Correo electrónico:juandukuen@gmail.com 
tradição britânica, em referência a uma prática específica do governo escolar: eleições para os capitães da escola. A partir de um trabalho de campo realizado entre 2016 e 2017 que incluiu entrevistas, observação participante e análise de documentos, as cartas candidatas para os capitães são analisadas para elucidar os significados que os alunos conceden a esta prática de representação escolar. Ressalta-se que liderança, habilidades de comunicação e antiguidade, constituem significados atribuídos às capitanias. Conclui-se relacionando-os a processos mais amplos de socialização politica juvenil nas classes altas, que levam a futuras linhas de pesquisa.

Palavras-chave: jovem, política, escola, classe alta, liderança, socialização política, disposições políticas (Thesaurus de Ciências Sociais da Unesco).

-1. Introducción: antecedentes y enfoque teórico. -2. Metodología. -3. Resultados. -3.a. House system, elecciones de captains y prefects. $-\mathbf{3 . b}$. Sentidos sobre las capitanías: un análisis de las cartas de los postulantes. -3.c. Liderazgo y escolaridad.-4. Discusión, conclusiones y perspectivas. -Lista de referencias.

\section{Introducción: antecedentes y enfoque teórico}

Este trabajo busca contribuir a las investigaciones sobre juventudes y política en la Argentina contemporánea (Chaves, 2010; Kriger, 2016, 2017; Vázquez, Vommaro, Núñez, \& Blanco, 2017; Vommaro, 2015) a partir del análisis de la socialización política estudiantil en un colegio de tradición británica, para clases altas, de la Ciudad de Buenos Aires, Argentina. Para ello, en este artículo analizo las cartas de postulación de los alumnos candidatos a capitanes de colegio en las elecciones anuales, con el objeto de elucidar los sentidos que ellos otorgan a esa práctica de representación política escolar, para luego establecer su relación con procesos más amplios de formación de disposiciones políticas en jóvenes de clases altas.

En las últimas décadas, en Argentina tuvo lugar un vasto desarrollo de investigaciones que abundan en el análisis de experiencias de participación estudiantil (Kriger \& Said, 2017; Larrondo, 2017; Núñez, 2013; Núñez \& Litichever, 2015), así como en partidos políticos y movimientos sociales en sectores populares y medios (Bonvillani, 2015; Vázquez et al., 2017). En contraste, el presente trabajo corresponde a una línea de investigación ${ }^{1}$ que establece vínculos con los estudios sobre elites y sectores favorecidos (Gessaghi, 2016; Tiramonti \& Ziegler, 2008; Ziegler \& Gessaghi, 2012) para contribuir al conocimiento de un dominio empírico poco trabajado en los estudios sobre juventudes y política: la socialización política de jóvenes de clases altas en colegios privados que no tienen instancias de militancia política partidaria ni Centro de Estudiantes (cf. Kriger \& Dukuen, 2012, 2014, 2017a, 2017b).

Siguiendo a Fillieule (2012), considero a la socialización política como «un proceso relacional y continuo de interiorización de esquemas de percepción y de acción relativos al mundo político o que forman parte de una relación política con el mundo social (...) sin resumirse en un dominio de conocimiento y de actividades especializadas: ella reenvía a un sistema de esquemas cognitivos y prácticos reconocidos o no como 'propiamente políticos' pero que tienen efectos de politización» (pp. 349-350). Asimismo, desde una perspectiva bourdiana poco trabajada en los estudios sobre juventudes y política (Kriger \& Dukuen, 2014), la socialización política es considerada como un proceso de interiorización en los agentes de disposiciones políticas (Bourdieu,1979), entendidas como sentidos in-corporados de pensamiento, percepción y acción, que actúan como resortes «subjetivos» de prácticas políticas, formadas en experiencias de socialización en diferentes dominios sociales (familiares, escolares, político-partidarias, etc.) y tramos de una trayectoria de clase o grupo (Dukuen, 2013).

1 Programa de Subjetividades políticas juveniles (CIS-Conicet/Ides) dirigido por la Dra. Kriger. 
A partir de esta perspectiva se puede comprender la dimensión política de la vida social como aquella cuyas prácticas producen, reproducen y/o transforman la distribución de los poderes sociales, es decir, de los capitales (económicos, culturales, sociales, simbólicos) entre grupos o clases (Dukuen, 2017). Esta definición «ampliada» de la dimensión política tiene varias ventajas: permite ir más allá de la política partidaria (incluyéndola); es productiva empíricamente tanto a «nivel micro» (interacciones familiares, escolares, etc.) como «macro» (organizaciones partidarias, Estado, etc.); permite comprender las dimensiones instituidas e instituyentes de las relaciones de poder; e invita a indagar esas relaciones en diferentes dominios de las prácticas, como el escolar.

En efecto, sin perder de vista que la escuela es una institución que «a partir de una determinada configuración socio-histórica, distribuye conocimientos y regulaciones morales que incluyen la dimensión política, y con ello, se conforma como un particular espacio de socialización y práctica política de los y las jóvenes» (Larrondo, 2017, p. 110), es central señalar que ella no es una ni la misma para los distintos grupos, clases y agentes, y que la relación que establece con ellos es desigual (Kriger \& Dukuen, 2017b). Esto significa que también los procesos de socialización política se juegan de diferente manera en la relación que se establece entre ciertas trayectorias de clase y formas específicas de escolaridad, entre las cuales interesa dilucidar las dirigidas a las y los jóvenes de sectores altos. Precisamente, a partir de establecer puentes con los estudios sobre educación de elites y clases altas en Argentina (Méndez, 2013, Tiramonti \& Ziegler, 2008; Ziegler \& Gessaghi, 2012) se advierte que con la aplicación sistemática de políticas neoliberales desde la década del 90, se produjo una creciente desigualdad social y fragmentación educativa entre las clases sociales que no ha sido revertida. Esta se expresa en una fuerte inversión de las familias de sectores medios-altos y altos en la oferta de educación privada del nuevo mercado escolar en el marco de las estrategias familiares de reproducción/transformación/ampliación de los capitales económicos, culturales y sociales (Martínez, Villa, \& Seoane, 2009) y en procesos de cierre social que tienden a una socialización «entre nos» (Gessaghi, 2016) cuya dimensión política es indagada en diversos trabajos referidos a continuación.

En artículos previos en colaboración (Kriger \& Dukuen, 2012, 2014) encontramos entre las y los estudiantes de clases altas mayores disposiciones a la participación política futura que en otras clases sociales. Además, mostramos cómo el haber compartido junto a sus padres los cortes de ruta contra el gobierno kirchnerista en el «conflicto del campo» ${ }^{2}$ se conformó como una experiencia clave de incorporación de disposiciones políticas que — en coincidencia con lo señalado por Vommaro y Morresi, 2015 - contribuyó a la conformación de la nueva derecha en Argentina (PRO) actualmente a cargo del Gobierno Nacional (Kriger, 2016). Asimismo en una investigación reciente (2014-2017)3 indagamos las diferentes modalidades en que la dimensión familiar interviene en la incorporación de disposiciones políticas en jóvenes escolarizados de diferentes clases sociales (Kriger \& Dukuen, 2017a, 2017b) y analizamos proyectos solidarios centrales en la propuesta institucional de escuelas de clases altas, mostrando que configuran prácticas de incorporación de esquemas morales que se vuelven disposiciones políticas (Dukuen \& Kriger, 2015, 2016).

En esta línea, para contribuir al conocimiento de los procesos de socialización política juvenil en estudiantes de clases altas, presento aquí hallazgos de un trabajo de campo etnográfico (Guber, 2005) en un colegio secundario de tradición británica de Ciudad de Buenos Aires, sobre la formación de disposiciones morales y políticas entre estudiantes de 4to y 5 to año del nivel secundario. En el marco de una reconstrucción de puntos de vista nativos sobre la elección de capitanes de colegio, en tanto práctica específica del gobierno escolar de las escuelas de tradición británica, las preguntas de

2 Refiere al conflicto entre el gobierno nacional de Argentina y los productores rurales en 2008 en torno al aumento del impuesto de retención a las exportaciones agrarias. Este episodio generó actos de protesta de las patronales rurales, que cortaron las rutas más importantes del país durante varias semanas, logrando el apoyo de las clases altas y gran parte de las medias. Los hechos tuvieron gran difusión mediática e impacto político y polarizaron a la opinión pública, conformando los cimientos del bloque político de centro derecha que llegó al poder en Diciembre de 2015 con la presidencia de Mauricio Macri (Kriger, 2016).

3 En el marco de proyecto Pict-ANPCyT 2012-2751 Dir. Dra. Kriger. 
investigación que guían este artículo refieren a cuáles son los sentidos que los candidatos estudiantiles les otorgan a las capitanías en sus cartas de postulación, y qué relaciones es posible establecer entre esas prácticas de formación de disposiciones políticas y procesos más amplios de socialización política en juventudes de clases altas.

El recorrido que propongo es el siguiente. Tras presentar las condiciones metodológicas del estudio, doy cuenta del modo de organización del alumnado en el colegio (House system) y describo las características de las elecciones de capitanes y representantes en el marco del gobierno escolar. Luego analizo las cartas de postulación para capitanes con el objeto describir los sentidos que dan a las capitanías, destaco su vínculo con el liderazgo, y con procesos más amplios de socialización política. Finalizo discutiendo los resultados en el marco de las conclusiones y señalo perspectivas abiertas en esta investigación.

\section{Metodología}

La escuela donde desarrollé la investigación — que llamaremos $\mathrm{T}$ - es la sede principal de una institución de tradición británica, mixta, de enseñanza bilingüe y laica con más de 50 años de trayectoria, que abarca los tres niveles educativos (jardín, primaria y secundaria) y cuya cuota mensual en el nivel secundario en 2017 fue de $\$ 14$ 000, es decir que está cerca de duplicar el salario mínimo, vital y móvil (\$8860). Se encuentra ubicada en la Comuna 13 de Ciudad de Bs. As., caracterizada por una población de clase media alta y alta (Vommaro \& Morresi, 2015).

Allí, durante los años lectivos 2016 y 2017 realicé un trabajo de campo de orientación etnográfica (Guber, 2005), ya que como señala Elsie Rockwell (2009) permite un estudio adecuado, en la escuela, de la producción y reproducción de las disposiciones. En ese sentido, en consonancia con indagaciones etnográficas (Darmon, 2013; Pasquali, 2014) preocupadas por describir «el proceso específico mediante el cual se configura un habitus determinado» descuidado por Bourdieu (1989) (Bernstein, 1996, p. 133; cf., Lahire, 2004) estudié, en la escuela, la producción de disposiciones morales y políticas entre estudiantes secundarios de 4to. y 5to. año, en el marco de una dilucidación de los puntos de vista de los «nativos» (Malinowski, 1987) es decir, de los sentidos que dan a esas prácticas quienes participan cotidianamente en ellas. Esta indagación implicó «observación participante» en diferentes actividades del nivel secundario del colegio (dictado de clases, reuniones del Consejo de convivencia, actos, elecciones de representantes y capitanes, actividades «extracurriculares»), análisis de documentos (Proyecto Institucional, cartas de postulación a capitanías, programas escolares), páginas web, charlas informales y 45 entrevistas con directivos, docentes, alumnos y preceptores ${ }^{5}$.

Con el objeto de comprender los sentidos que los candidatos dan a las capitanías, en este artículo presento análisis de extractos significativos del total de las cartas de postulación (11) presentadas por estudiantes de 4to año del colegio secundario (16 años de edad) para las elecciones a capitanes de colegio. Utilizo para ello la perspectiva propuesta por Bourdieu (1982) consistente en detectar y analizar sentidos que indiquen relaciones de reconocimiento y legitimación entre los agentes. Así mismo, para ampliar el fundamento de la indagación y dar cuenta del modo de organización del alumnado y de las elecciones, retomo extractos de documentos institucionales, de entrevistas con estudiantes y directivos, así como observaciones realizadas en el trabajo de campo.

4 Además de tomar la información relativa a la cuota del colegio y compararla con el salario mínimo, vital y móvil, teniendo en cuenta que nos vimos imposibilitados de recolectar datos sobre nivel de ingresos, siguiendo a Bourdieu (1979, p. 128) consideramos un buen indicador de la condición de clase de los alumnos el capital cultural del padre medido por el máximo nivel de escolarización. En el caso de las y los alumnas/os de 4to. y 5to. año del colegio (los dos años finales), aplicamos un cuestionario escrito individual autoadministrable de 30 ítems que incluía una pregunta sobre el máximo nivel educativo alcanzado por el padre. Sus resultados muestran un alto porcentaje de escolarización de nivel universitario que reenvía a los sectores medios-altos y altos en Argentina: el 62,8 por ciento de los padres de los y las alumnas/os posee estudios terciarios/universitarios completos; 20,9 por ciento, posgrado; el 11,6 por ciento, secundarios; y el 4,7 por ciento, primario. [Datos provenientes de un cuestionario aplicado en 5 colegios de diferentes clases sociales en el marco del proyecto Pict 2012-2751 (Dir: Dra Kriger). Ver al respecto Kriger y Dukuen (2017a y 2017b)]. Sobre la estructura de clases en Argentina ver Benza (2016).

5 Para proteger la identidad del colegio y de los entrevistados modifiqué sus nombres. Esbozo un ejercicio de reflexividad sobre esta experiencia etnográfica en Dukuen (2017). 
SOCIALIZACIÓN POLITICA JUVENIL EN UN COLEGIO DE CLASES ALTAS (BUENOS AIRES, ARGENTINA)

\section{Resultados}

\section{3.a House system, elecciones de captains y prefects}

En términos de una indagación más general sobre los colegios de tradición británica alrededor del mundo, pude saber que las y los estudiantes son distribuidos en houses, a partir de lo que llaman «House system». Buscando en las páginas web de colegios de estas características, encontré una explicación breve sobre esta forma tradicional de organización escolar:

The house system is a traditional feature of schools in the English-speaking world, particularly in Commonwealth countries, originating in England. The school is divided into subunits called «houses» and each student is allocated to one house at the moment of enrollment. Historically, the house system was associated with established public schools in England, especially full boarding schools, where a «house» referred to a boarding house at the school. In modern times, in both day and boarding schools, the word «house» may refer only to a grouping of pupils, rather than to a particular building. Each house will usually also be identified by its own symbol, logo, or (...) different colours. (House system, 2017)

Cuando consulté con la directora del Colegio $\mathrm{T}$ la historia de esta forma de organización, para darme una idea que me pudiera ser familiar hizo referencia a la exitosa saga de novelas Harry Potter, de J. K Rowling, a las houses de la Hogwarts School donde transcurre la trama, y me envió un link de una enciclopedia web. También otros integrantes de la institución, algunos de los cuales eran ex alumnos del colegio T o de similares, me ayudaron a comprender el «House system».

La organización del alumnado en términos de houses con sus respectivos captains aparece de forma recurrente en las instituciones educativas de tradición británica en Argentina. Ella forma parte de un ethos compartido, de un mundo común de sentido que se observa en los lazos que existen entre estos colegios en el marco de instituciones como ADE (Asociación Deportiva Estudiantil), E. S. S. A. R. P. (English Speaking Association of the River Plate), L. A. H. C. (Latin American Heads Conference) y E. S. U. (English-Speaking Union). En el caso del colegio T, al ingresar a la institución cada alumno es asignado a una de las tres houses representadas por los tres colores de la escuela. Las houses se asignan por herencia familiar o por distribución equitativa de integrantes. En el reglamento de capitanes se señala que «cada house está conformado por un grupo de alumnos de primaria y secundaria integrados verticalmente por el objetivo de trabajar juntos en distintas actividades en un marco de sana competencia. Sus miembros pertenecen durante toda su escolaridad al mismo house guardando entre sí compañerismo, lealtad, respeto y solidaridad». A lo largo del año cada house acumula puntajes en relación a cada actividad o evento cultural y deportivo (Sports) en el que participe. Al finalizar el año se suma el puntaje obtenido y el house que obtuvo mayor puntaje se hace acreedor del shield, escudo en el que irá grabado el nombre del capitán.

La participación estudiantil forma parte del gobierno escolar, entendido como «las reglas de juego explícitas e implícitas que son capaces de generar ciertas condiciones para la participación, - y eventualmente la politización - a la vez que dichas estructuras son resultado de ella» (Larrondo, 2017 p. 121) y está encuadrada en el «Aspecto afectivo-social y cívico» del Proyecto Educativo Integral (PEI) del colegio, donde «la exploración de la subjetividad propia y el ejercicio de la participación social aumentan los niveles de responsabilidad y compromiso». Esta propuesta se lleva adelante mediante proyectos tales como «liderazgo participativo, asambleas escolares, elecciones de representantes de curso y líderes estudiantiles, proyectos institucionales colaborativos, mindfulness y el proyecto de educación en valores» (PEI).

Tradicionalmente, los capitanes eran elegidos por las autoridades en base al criterio de buen rendimiento escolar. A partir de la asunción de la directora actual en 2010 y con el apoyo de otros colegas se implementó el sistema de elección mediante votación en el que participan los alumnos.

La elección de capitanes (captains) y de representantes de curso (prefects) no se enmarca solo en prácticas consuetudinarias, sino que además se encuentra objetivada en el reglamento al que hacíamos 
referencia. Si hacemos un análisis comparativo, los prefects operan entre sí en un nivel «horizontal», en la medida en que no hay relaciones de subordinación entre los representantes de diferentes cursos, como si lo hay en el caso del Equipo de capitanes. El mandato de los prefects es de alcance interno, es decir, que refiere solo a las relaciones de cada curso con los docentes, el Consejo de convivencia y el cuerpo directivo.

En el trabajo de campo pudimos observar que la elección del «Equipo de capitanes (aptainsLeadership)» tiene mayor alcance e importancia a nivel institucional. ¿Cómo se produce «la elección de los elegidos» (Bourdieu \& Passeron, 1964)? Los capitanes son elegidos entre alumnos de $4^{\circ}$ de nivel secundario para ejercer sus cargos al año siguiente, en su último año escolar $\left(5^{\circ}\right)$. En julio, aproximadamente - ya que las fechas varían levemente año a año- durante una semana las y los estudiantes pueden presentar las cartas de postulación a la Directora. Un mes después, una vez aceptadas las postulaciones, pueden comenzar la «campaña», que se extiende entre agosto y la primer semana de noviembre, aproximadamente, y finaliza una semana antes de la votación. Ella consiste generalmente en publicitar la postulación, realizar afiches y pegarlos en espacios designados y así comunicar sus propuestas a los demás estudiantes. Unos días antes de las elecciones, en un acto de «cierre de campaña» ante los alumnos del colegio de 7 mo. grado a 5to. año, los postulantes a capitanes dan un discurso donde cuentan las razones por las cuales se postularon y sus objetivos, que generalmente retoma lo señalado en las cartas de postulación. Los dos días en que se realizan las elecciones, a partir de 7 mo grado «votan, los alumnos, los docentes y los directivos. Se tiene en cuenta la tendencia del alumnado y la opinión del cuerpo docente. Deciden los coordinadores y directores», señala el reglamento. La votación se realiza en la Biblioteca donde la bibliotecaria, se encarga de realizar las boletas, preparar la urna y supervisar con mucha dedicación el proceso de elecciones, cuyos resultados anuales guarda en su computadora. En analogía con las elecciones nacionales, se refirió a si misma como una «presidenta de mesa» que va saldando las dudas de las y los alumnos, sobre todo de los que votan por primera vez en séptimo grado. En los días de elecciones, estudiantes, docentes y directivos se van acercando a la biblioteca, y en el escritorio de la bibliotecaria encuentran la urna y las boletas: toman la boleta que corresponde (son tres, una por house) según su pertenencia a una house, van hacia una suerte de «cuarto oscuro», tildan las opciones de capitanías y representantes de curso y depositan su voto en la urna. Al finalizar, los votos son contados por directivos y docentes y en los últimos años por tres fiscales alumnos que se ofrecen para tal fin. Luego, se comunican los resultados sobre los capitanes y representantes de curso electos.

En el reglamento, se indica que el «Equipo de capitanes» consiste de un grupo de alumnos del último año escolar, «elegidos por sus méritos, cualidades específicas y alto perfil para desempeñar determinadas funciones dentro de la institución». A continuación se realiza un señalamiento que es de importancia para nosotros en la medida en que da la pauta de que desde el punto de vista institucional se proyecta que este tipo de prácticas puedan estar en la base de prácticas posteriores: «el aprovechamiento que los capitanes hagan de este honor podrá ser de importancia para su vida futura, transfiriendo la experiencia que adquieran a través de este rol a otros ámbitos de su vida personal, académica y laboral», señala el Reglamento de capitanes. En esa línea, por ejemplo, hemos observado que algunos jóvenes egresados de colegios de tradición británica que han sido capitanes, los hacen constar en su curriculum laboral.

El Equipo de capitanes del colegio se compone de la siguiente manera: Capitán General (School Captain-School Leadership), Capitán de Deportes (Games Leadership), Capitán de Acción Social (Social Work Leadership). Capitanes de Houses: Capitán de A House, Capitán de B House y Capitán de C House.

Según consta en el reglamento sus objetivos son: fomentar y fortalecer el espíritu del colegio; estimular la integración de los distintos sectores del colegio; representar al colegio en todas las áreas (cultural, deportiva y social); ser líderes positivos que demuestren actitudes de compromiso ante la institución; demostrar ansias de superación personal y académica; ser miembros del Consejo de Convivencia. 
Hay una relación vertical entre ellos: el Capitán General, coordina a los Capitanes de Deportes y Acción Social. A su vez ellos coordinan en cada área a los capitanes de las houses.

El Capitán del colegio representa a los alumnos en eventos académicos y sociales; es su vocero en cuestiones de orden general; actúa de enlace entre la dirección y los alumnos en la organización de eventos; orienta la función del resto de los capitanes, manteniéndose al tanto de los distintos proyectos; participa en los assemblies y organiza eventos con los distintos sectores del colegio.

El Capitán de Deportes representa al alumnado ante las autoridades del colegio y el coordinador de Educación Física; coordinar las tareas deportivas de los capitanes de houses; actúa de enlace entre el coordinador de Educación Física y los alumnos en la organización de eventos deportivos; representa al colegio en eventos deportivos intercolegiales y fomentar el espíritu deportivo en todas las actividades del área

El Capitán de Acción Social representa al alumnado ante las autoridades del colegio y coordina las tareas de acción social de los capitanes de houses; actúa de enlace entre las autoridades y los alumnos en la organización de eventos de acción social; representa al colegio en eventos sociales.

Los capitanes de houses representan a los miembros de cada house; organizan y participan de eventos culturales, deportivos y sociales; fomentan la comunicación visual a través de las carteleras; reúnen a los miembros de su house en forma general o parcial para fomentar la comunicación personal y organizan encuentros con alumnos de los sectores de primaria y jardín para fomentar el espíritu de integración y pertenencia.

\section{3.b Sentidos sobre las capitanías: un análisis de las cartas de los postulantes}

Como señalé más arriba, en el marco de proceso anual de elección de capitanes, los alumnos de 4to año que desean postularse, deben presentar una carta a la Directora donde expresen sus razones y hacen sus propuestas. Si se acepta la postulación, pueden iniciar su «campaña» que consiste tanto en charlar informalmente con sus compañeros y docentes, la preparación de afiches propagandísticos y preparar el discurso para leer en el cierre de campaña de capitanías. La carta de postulación enviada a la Directora es central, porque en la mayoría de los casos es la base del discurso que darán ese día - como pude observar en el cierre de campaña-, donde le dicen a sus pares, a directivos y docentes, cuales son sus objetivos y por qué deberían votarlos.

Las cartas se configuran en torno a argumentar las razones por las cuales se postulan y presentar cuales son los principales objetivos a alcanzar en caso de que accedieran a ser capitanes. Una primera lectura evidencia que es recurrente en los postulantes a capitanes (sean de la categoría que sean), hacer referencia a su antigüedad en el colegio: «Formo parte de esta familia tan cálida desde que tengo 3 años» (J, Capitanía de house); «Desde que era muy chica siempre quise ser captain» (K, Capitanía de Acción Social); «Ser capitana del colegio ha sido como un sueño desde que entré a primaria» (M, Capitanía General). Esta apelación, aparece en las cartas más que todo como una forma de legitimación basada en el conocimiento y la ligazón afectiva con la institución (referencias al deseo y honor de pertenecer, a la admiración de capitanes anteriores ${ }^{6}$, a la institución como familia). Incluso en algunos postulantes esto opera por la negativa, dando por supuesto el valor de la antigüedad y por lo tanto teniendo que justificarse estratégicamente ante la falta, lo cual es recurrente en aquellos que ingresaron al colegio en el secundario. Una postulante señalaba «No estoy en el colegio desde muy chica ni tampoco siempre quise ser capitana pero desde el año pasado que es algo que me interesa» (L, Capitanía General).

6 Algunos testimonios: «Desde muy pequeña veía a los capitanes de secundaria y me preguntaba a mí misma si era posible ser votada como capitana general algún día. Siempre los observaba con admiración, soñando ser como ellos» (M, Capitanía General). «Otra razón por la cual me postule fue haber visto el compromiso y compañerismo de los capitanes del 2014, ver en detalles, por ejemplo, cuando llevaron pinturas de colores a los Sports como motivaban a los más chicos a competir fue realmente conmovedor. También me gustó la forma en que la que hicieron distintos discursos y usaron responsablemente su distinción.» (E, Capitanía House). 
Por contraste, pudimos observar en las entrevistas que otros alumnos específicos se consideraban (auto) excluidos: es el caso de las dos becarias de la institución (ampliar en Kriger \& Dukuen, 2017b) que habían ingresado en 4to. año. En una entrevista una de ellas $(Z)$ señalaba:

«Z - No, no, no me presenté para nada () era el año pasado que teníamos que ponernos, que postularnos. Primero no podía porque yo soy nueva, el año pasado, y ahí entré y tenía que ser alguien que esté desde los cinco, o sea que hubiera entrado por ejemplo en primaria. Entrevistador - Ah... eso es parte del reglamento...

$Z$ - Es algo así pero yo tampoco soy mucho de hablar con la gente entonces tenía que ser alguien que haya ido a todos los campamentos, que hable con los cursos, y yo era nueva y no me animaba.» (Z, Becaria del Colegio)

Justamente por que las prácticas no se producen por un ajuste mecánico a las reglas (Bourdieu, 1987) cabe indicar que el reglamento nada dice sobre la necesidad de haber ido al colegio los cinco años, ni tampoco sobre estar allí desde la primaria. Por eso mismo, el hecho de creer que «es algo así», es un analizador de cuales son los sentidos comunes que circulan y las fronteras simbólicas que se instituyen entre quienes son o no los postulantes legítimos para las capitanías, que exceden a los reglamentos. En ese sentido, la antigüedad, la participación de actividades específicas y el conocimiento «del colegio» se volvían centrales en la forma de legitimar la posibilidad de presentarse, es decir que relacionalmente se constituían como un capital simbólico, en este caso, como un capital social que es percibido con reconocimiento (Bourdieu, 1979). Quien fue elegido finalmente capitán general señala en su carta:

«Vengo al colegio desde los 3 años. Fui prefect/delegado de la clase a partir de segundo año de la secundaria hasta el día de hoy. Esas pueden que sean una de las razones por las que quiero ser capitán. Siento que conozco mucho al colegio, no solo en cuanto a la estructura sino que también a los profesores, alumnos, los objetivos del colegio y el mismo en sí. En cuanto a que soy prefect/delegado hace 3 años seguidos siento que es algo importante ya que pasé por la experiencia de ser, en algún sentido, el 'capitán'de la clase.» (A, Capitanía General)

En este caso hay dos principios de legitimidad que se esgrimen. El primero relativo a la antigüedad que le brinda un conocimiento del colegio en todos los niveles. El segundo, a que viene ocupando cargos de prefect en los últimos tres años, lo cual le daría saberes específicos, sobre las actividades de representación. Él mismo realiza la analogía entre ambos niveles, diciendo que ha sido el «capitán» de la clase. En su presentación de sí, esta estrategia le permite, «ponerse en regla» (Bourdieu, 1987), es decir, «cumplir» con criterios centrales que la institución ha señalado para elegir capitanes: trayectoria personal, liderazgo positivo, comunicación con otros sectores del colegio, capacidad de incorporar la función de capitán. En ese sentido, en la mayor parte de las cartas se observan referencias que ligan las prácticas relativas al ejercicio de las capitanías, con nociones como «líder» y «liderazgo», en las cuales me quiero detener para reconstruir los sentidos atribuidos. Veamos algunos ejemplos:

«Sería de gran ayuda que nosotros, los líderes, los incentivemos a comprometerse con algunos aspectos académicos y también personales (...) Mi principal objetivo y motivo por el cual quiero ser captain es para aumentar la integración de los mas chicos con los mas grandes y poder ayudarlos en lo que sea que necesiten.» (H, Capitanía General)

"Los motivos para ello son varios, entre los cuales está la admiración que yo sentía al mirar a los capitanes cuando era un niño, los veía como líderes y personas muy correctas. Mi principal objetivo se concentra en tratar de integrar a todos los cursos de la secundaria de manera tal que todos puedan relacionarse entre sí.» (F, Capitanía de house)

«Soy una gran admiradora de las mujeres lideres, y me gustaría ser la de White. Si soy elegida voy a servir al bienestar de cada integrante de White. Quiero darles el sentido de unión, y el de amor por su color.» (J, Capitanía de house)

¿Cuáles son los sentidos que los postulantes atribuyen a nociones como «líder» y «liderazgo»? La tarea de llevar adelante la integración y la unión del alumnado todo, en el caso del Capitán General, y la integración de los alumnos de las houses en las capitanías correspondientes, aparecen como sentidos 
centrales de las prácticas. La referencia a dar «el sentido de unión, y el de amor por su color» es un punto clave a fomentar porque las houses compiten entre sí en diferentes actividades en el año. En los Sports y en diferentes actos, por ejemplo, las diferentes houses cantan canciones de aliento a sus colores y se ve sobre todo en «los más chicos» el entusiasmo por cantar más fuerte. En ese sentido es importante tener en cuenta que la experiencia de pertenencia a una house, atraviesa toda la escolarización de los alumnos, e implica el uso de vestimentas específicas según color, así como prácticas recurrentes, tales como las competencias culturales y deportivas a las que hacíamos referencias (rugby masculino, hockey femenino, atletismo) y sus concomitantes entregas de distinciones. Es decir, no son prácticas fragmentarias y esporádicas, sino que mantienen una regularidad en el tiempo escolar y algunas en particular pueden ser comprendidas como ritos de institución (Bourdieu, 1982) que unen y separan al mismo tiempo y establecen una frontera entre quienes pertenecen y quienes no: es el caso del acto de entrega de los distintivos de colores a los nuevos integrantes de cada house - los que ingresan en primaria y los que viene de otros colegios - el cual es organizado por los capitanes de quinto año, en marzo; y también lo es el acto de entrega del shield, en noviembre/diciembre, en el cual se despide a los captains salientes, y se da la bienvenida y los distintivos a los nuevos prefects y captains. Este conjunto de prácticas operan simbólicamente en varios niveles: a) distribuyen a las y los estudiantes en las diferentes houses, en las cuales van a transcurrir su escolaridad, b) establecen jerarquías entre el equipo de capitanes y el resto de alumnas y alumnos, ya que invisten simbólicamente a quienes son capitanes frente a quienes no lo son, otorgándoles atribuciones que los demás no tienen c) establece jerarquías entre los capitanes, según el orden vertical que los coordina. Además, en estas experiencias los estudiantes incorporan disposiciones y esquemas de pensamiento, percepción y acción, es decir, un ethos específico que configura el punto de vista nativo y los principios de visión y división que operan en ese espacio social escolar.

El «liderazgo» se relaciona así mismo con «motivar» a los demás, especialmente a colaborar y competir, como se observa aquí:

"Otra idea que me atrae es la de integración. Me parece cautivador el hecho de que a la hora de competir para tu house, por ejemplo, los niños de 7 años colaboren con los de 15 de una forma más directa y viceversa, en la cual el de 15 puede ayudarlo ya sea motivándolo o preparándolo. De esta forma, se ejercita el trabajo en grupo y aumenta la eficacia de cada integrante porque, como se sabe, cuando en un grupo se establece afinidad, aprecio, apoyo, se valora la actuación de cada uno, festejando logros y alentando al chico ante fracasos con el objetivo de motivarlo a seguir intentando.» (B, Capitanía Deportes)

Al mismo tiempo, la importancia de la comunicación del capitán con el alumnado se vuelve central para la resolución de problemas y para el impulso de proyectos. Por ejemplo, el Capitán General señala:

«Yo me ofrezco a ser quien le transmita la confianza suficiente a todos los alumnos para que ellos me transmitan el problema que tengan y buscar la solución al mismo. Puede que suene poco importante pero desde el lado del alumno yo creo que es indispensable. (...) También pienso que como capitán tomaría en cuenta cualquier idea para un nuevo proyecto que un alumno me transmita y si esta idea es adecuada y posible intentar cumplirla.» (A, Capitanía General)

En un sentido similar, apunta la carta de quien fue finalmente elegida Capitán de una de las houses:

«Personalmente, me siento una persona muy capaz de llevar a cabo un liderazgo que pueda beneficiar al colegio, animándome a presentar y empezar nuevos proyectos. Uno de mis mayores objetivos si llegara a ser capitana sería favorecer a los alumnos, que ellos puedan decirnos lo que necesiten y que podamos tener una buena comunicación entre todos. El progreso social es muy importante ya que aprender a convivir es clave para una buena educación. Así mismo, me gustaría funcionar como una intermediaria entre los alumnos y las autoridades.» (E, Capitanía de house). 
En síntesis: la antigüedad como legitimación; la comunicación con alumnos y la mediación con docentes; la motivación para producir identificación e integración afectiva con la institución y/o con las houses y promover el interés por la competencia - donde cada cual juega su rol - y el impulso de proyectos que tengan en cuenta las propuestas de las y los demás estudiantes, son algunos de los sentidos recurrentes que los postulantes atribuyen el ejercicio de una capitanía, comprendida en muchos casos en términos de «liderazgo». Estás prácticas, valoradas por la institución en sus proyectos y por las y los alumnos en las cartas y entrevistas, componen un ethos que no gira sobre sí mismo, sino, que en un movimiento centrífugo y centrípeto (Merleau-Ponty, 1945), reenvía a otros dominios de las prácticas (especialmente al Management empresarial) con las cuales mantiene afinidad de sentido. Sobre este punto me detendré a continuación.

\section{3.c Liderazgo y escolaridad}

Aunque la formación de líderes no sea uno de los objetivos institucionales del colegio, al informar sobre la ceremonia de entrega de House Colours, en su página web señala: «Incentivamos, a través de la conformación de grupos por house, el liderazgo participativo, la construcción colectiva en la toma de decisiones y la posibilidad de hacer junt@s». En el trabajo de campo pude observar que si bien hay diferencias entre docentes, directivos, preceptores y estudiantes sobre la significación del «liderazgo» y su vínculo con las capitanías, la noción forma parte de un mundo común de sentido que atraviesa la vida escolar.

La categoría de «liderazgo» forma parte de la gramática managerial (Luci, 2012) que compone el «nuevo espíritu del capitalismo» (Boltanski \& Chiapello, 2002), «conjunto textual con vocación preformativa» orientado a naturalizar una perspectiva particular sobre el mundo del trabajo y a prescribir determinadas maneras de pensar y actuar con base en «valores como la autonomía, la creatividad y la personalización de las relaciones en el trabajo» (Mazzini, 2013, pp. 14-15, 17). Luci (2012) señala que «valoran especialmente las actitudes tendientes a generar la identificación, los vínculos sólidos y el buen clima en el espacio interno de la empresa ( ) los manager entrevistados dicen implementar diversas estrategias para motivar y comprometer a sus subordinados» (p. 180).

En tanto ethos que se ha expandido a otros dominios de las prácticas como el escolar y el político, el «liderazgo» que los capitanes tienden a encarnar, encuentra afinidad de sentido con la gramática managerial (motivar, producir identificación) y reenvía a una serie de actividades escolares. Un caso ejemplar es la «Capacitación en liderazgo en valores» que la escuela llevó adelante el año anterior al trabajo de campo con los alumnos de cursos superiores. Para ello contrató a la consultora experiencia líderes, conformada por profesionales en management, educación, liderazgo político y capacitación multimedia. La consultora exhibe en su página web sus trabajos de formación en liderazgo con instituciones educativas de clases altas y empresas y presenta su programa de «liderazgo político en valores», el cual tiene como principal participante al partido político PRO y al Ministerio de Ambiente y Espacio público de la Ciudad de Buenos Aires. En relación con ello, las convivencias grupales escolares, que realizan los cursos cada año, también se vuelven espacios educativos para aprehender estos valores: una de ellas, por ejemplo, se avocó al «liderazgo en valores». Para la institución «fue una jornada colmada de entusiasmo e intenciones claras orientadas al liderazgo positivo, el cual especialmente espera a nuestros alumnos de Cuarto Año en la próxima actividad de campamento estudiantil y en las Capitanías». Además, las y los estudiantes son invitados a participar de otros programas que operan en el mismo sentido, tales como «Socios por un día» organizado hasta por la ONG Junior Achivement, una de las mayores ONG a nivel global de educación económica, emprendedurismo y formación para niños, niñas y jóvenes, el cual en las entrevistas fue muy valorado por ellos, porque les permitía tener una experiencia previa en actividades profesionales sobre las que iban a realizar sus estudios universitarios. 


\section{Discusión, conclusiones y perspectivas}

En este artículo presenté hallazgos de un trabajo de campo de orientación etnográfica realizado en un colegio de tradición británica para clases altas de la Ciudad de Buenos Aires. Los resultados a los que he arribado permiten responder a las preguntas de investigación presentadas en la introducción relativas a cuáles son los sentidos que los candidatos estudiantiles les otorgan a las capitanías en sus cartas de postulación, y qué relaciones es posible establecer entre esas prácticas de formación de disposiciones políticas y procesos más amplios de socialización política en juventudes de clases altas.

Con respecto a los sentidos que los postulantes a capitanes dan a las capitanías, el análisis de las cartas permitió observar que la comunicación con los alumnos y la mediación con los docentes; la motivación para producir identificación e integración afectiva con la institución o con las houses; el promover el interés por la competencia; y el impulso de proyectos participativos, son sentidos que atribuyen a las capitanías comprendidas como «liderazgo», los cuales confluyen con la gramática managerial (Luci, 2012), como se observa en el apartado anterior. Esos sentidos nativos se comprenden especialmente en el marco del sistema de relaciones que implica el House system, tal como aquí fue descrito: una forma de organización del alumnado de los colegios de tradición británica que ordena la participación de las y los estudiantes en los eventos deportivos y culturales, así como los rituales que organizan la temporalidad escolar, a partir de su pertenencia a alguna de las houses. El House system establece prácticas de integración moral y afectiva entre compañeros de cada house, así como con el colegio en general, en una forma específica de socialización «entre nos» que permite incorporar las disposiciones necesarias para transitar esa escolarización. En ese sentido, los candidatos a capitanes reconocen que la integración del alumnado es una de sus tareas claves como líderes.

Ahora bien, los hallazgos de esta investigación basada en un estudio de caso, si bien no son generalizables a otras formas de escolarización - esa es su limitación-, desde una perspectiva bourdiana de orientación etnográfica buscan contribuir tanto al conocimiento de la socialización política juvenil en colegios de tradición británica para clases altas, como a tender puentes con los estudios sobre elites y sectores favorecidos. Esto permite complejizar la comprensión sobre la formación de disposiciones políticas en clases altas (Kriger \& Dukuen, 2014; 2017a, 2017b), poco trabajada por los estudios sobre juventudes y política en Argentina, cuyo foco han sido los sectores populares y medios, como señalamos en la introducción.

En ese camino, me interesa discutir perspectivas y alcances de esta investigación relativas al vínculo que las prácticas de formación escolar de disposiciones morales y políticas (un ethos) aquí analizadas, tienen con procesos más amplios de formación de socialización política.

En un primer nivel de análisis, el proceso de elecciones para las capitanías que forma parte del gobierno escolar es formador de disposiciones políticas en la medida en que desde séptimo grado implica aprehender prácticas de elección de representantes mediante el voto, configuradas en analogía con la elección de representantes para cargos políticos en una democracia liberal tal como se práctica en Argentina, donde se puede votar a partir de los 16 años. Es decir, que estas prácticas son ejercicios de formación de ciudadanía, siguiendo en esto una de las tareas fundamentales de la escuela en los estados liberales a partir de la modernidad (Kriger, 2010).

En un segundo nivel de análisis, las elecciones para capitanes y la pertenencia a houses, operan en las distribución de los poderes en el gobierno escolar: establecen jerarquías entre el equipo de capitanes y el resto de los alumnos, ya que invisten simbólicamente a quienes son capitanes frente a los que no lo son - y los que nunca lo serán, como las becarias-, otorgándoles atribuciones que los demás no tienen; y establece jerarquías entre los capitanes, según el orden vertical que los coordina. Como pude observar en el trabajo de campo, estás prácticas contribuyen a producir subjetivamente en postulantes y capitanes elegidos el reconocerse y ser reconocidos como «líderes», incorporando disposiciones y esquemas de pensamiento y acción específicos, «en el sentido de capacidad socialmente reconocida, de atributos y atribución estatutarios» (Bourdieu, 1979, pp. 465-466) entre los cuales se destacan los relativos a hablar en público; organizar diferentes eventos motivando y coordinando a 
demás estudiantes y generar sentido de pertenencia y tomar decisiones en el consejo de convivencia, los cuales valoran para su futuro como estudiantes universitarios y profesionales.

En un tercer nivel de análisis, que se configura como una de las futuras líneas de investigación, es menester indagar la formación en liderazgo que cristaliza el ethos proveniente del Management empresarial, vuelto un capital simbólico eficiente en diversos dominios de las prácticas, como el escolar y el político. Un hecho sintomático es que si bien la formación de líderes no es uno de los objetivos del Colegio $\mathrm{T}$, la noción forma parte de un mundo común de sentido que atraviesa la vida escolar en afinidad con la gramática managerial. En relación con el dominio político, un ejemplo de confluencia se observa en que el partido político PRO — representante de la centroderecha a cargo del gobierno nacional en Argentina - recluta militantes en juventudes con experiencias en emprendedorismo y voluntariado en escuelas de clases altas y universidades privadas, donde el liderazgo se vuelve una pieza clave en la dirección de equipos (Grandinetti, 2015; Vommaro \& Morresi, 2015).

Al mismo tiempo y como perspectiva a indagar en investigaciones futuras, estos señalamientos indican que las prácticas de educación moral escolar, relativa aquí a las houses y capitanías escolares, y en otros colegios a las actividades solidarias (Dukuen \& Kriger, 2015, 2016), contribuyen a dotar a ciertos estudiantes de competencias sociales de liderazgo que, mediante activaciones en socializaciones políticas posteriores pueden transfigurar en una acumulación de capital moral (Wilkis, 2013 ) potencialmente reconvertible en capital militante (Poupeau, 2007).

El conjunto de los hallazgos y perspectivas de investigación señaladas en este artículo pretenden así contribuir a la comprensión de diferentes dimensiones de la socialización política de las y los jóvenes de clases altas en Argentina, en el marco de amplios y heterogéneos procesos de politización juvenil en las naciones contemporáneas (Kriger, 2014, 2016).

\section{Lista de referencias}

Benza, G. (2016). La estructura de clases argentina durante la década 2003-2013. En G. Kessler (Comp.), La sociedad argentina hoy: radiografía de una nueva estructura, (p. 111-140). Buenos Aires: Siglo XXI.

Bernstein, B. (1996). Pedagogía, control simbólico e identidad. Madrid: Morata.

Boltanski, L., \& Chiapello, E. (2002). El nuevo espíritu del capitalismo. Buenos Aires: Fondo de Cultura Económica.

Bonvillani, A. (2015). Callejenado la alegría y también el bajón. Córdoba: EGE.

Bourdieu, P. (1979). La distinction. Paris: Minuit.

Bourdieu, P. (1982). Ce que parler veut dire. Paris: Fayard.

Bourdieu, P. (1987). Choses dites. Paris: Minuit.

Bourdieu, P. (1989). La noblesse d'État. Paris: Minuit.

Bourdieu, P., \& Passeron J-C. (1964). Les héritiers. Paris: Minuit.

Chaves, M. (Coord.) (2010). Estudios sobre Juventudes en Argentina. La Plata: Edulp.

Darmon, M. (2013). Classes préparatoires. La fabrique d'une jeunesse dominante. París: La Découverte

Dukuen, J. (2013). Habitus y dominación: para una crítica de la teoría de la violencia simbólica en Bourdieu. (Tesis de Doctorado, Facultad de Ciencias Sociales). Universidad de Buenos Aires, Buenos Aires, Argentina.

Dukuen, J. (2017). La elección de los elegidos: sentidos prácticos sobre las capitanías en jóvenes estudiantes de una escuela secundaria de clases altas. Actas de las XII Jornadas de Sociología, Facultad de Ciencias Sociales, Universidad de Buenos Aires, Buenos Aires, Argentina.

Dukuen, J., \& Kriger, M. (2015). Educación moral y formación de disposiciones políticas en jóvenes escolarizados de clases altas. Actas del V Congreso Nacional de Investigación y Práctica Profesional en Psicología, Facultad de Psicología, Universidad de Buenos Aires, Buenos Aires, Argentina.

Dukuen, J., \& Kriger, M. (2016). Solidaridad, esquemas morales y disposiciones políticas en jóvenes 
de clases altas: hallazgos de una investigación en una escuela del conurbano bonaerense (20142015). Revista Astrolabio. Nueva Época, 15, 311-339.

Fillieule, O. (2012). Travail, famille, politisation. En I. Sainsaulieu, \& M. Surdez (Eds.), Sens politiques du travail, (pp. 345-357). Paris: Armand Colin.

Gessaghi, V. (2016). La educación de la clase alta argentina. Buenos Aires: Siglo XXI.

Grandinetti, J. (2015). Mirar para adelante: tres dimensiones de la juventud en la militancia de Jóvenes PRO. En G. Vommaro, \& S. D. Morresi (Org.), Hagamos equipo: la construcción de PRO y la renovación de la centroderecha argentina, (pp. 231-263). Los Polvorines: UNGS.

Guber, R. (2005). El salvaje metropolitano. Buenos Aires: Paidós.

House system. (2017). The House System at the Berlin British School. Recuperado de: http://www. berlinbritishschool.de/house-system/

Kriger, M. (2010). Jóvenes de escarapelas tomar. La Plata: UNLP.

Kriger, M. (2014). Politización juvenil en las naciones contemporáneas: el caso argentino. Revista Latinoamericana de Ciencias Sociales, Niñez y Juventud, 12(2), 583-596. doi:10.11600/169271 $5 x .1225310314$

Kriger, M. (2016). La tercera invención de la juventud. Buenos Aires: GEU.

Kriger, M. (Dir.) (2017). El mundo entre las manos: juventud y política en la Argentina del Bicentenario. La Plata: UNLP.

Kriger, M., \& Dukuen, J. (2012). Clases sociales, capital cultural y participación política en jóvenes escolarizados. Una mirada desde Bourdieu. Question, 1(35), 317-327.

Kriger, M., \& Dukuen, J. (2014). La política como deber: un estudio sobre las disposiciones políticas de estudiantes argentinos de clases altas (Buenos Aires, 2011-2013). Persona y Sociedad, $X X \operatorname{VIII}(2), 59-84$

Kriger, M., \& Dukuen, J. (2017a). ¿En el nombre del padre?: dimensión familiar y disposiciones políticas en jóvenes estudiantes de una escuela de clases altas de la Ciudad de Buenos Aires». Revista Última Década, 46, 151-168.

Kriger, M., \& Dukuen, J. (2017b). Haciendo de la necesidad virtud: socialización política y herencia familiar entre becarias de un colegio de clases altas. Revista Pilquen, 20(3), 67-81.

Kriger, M., \& Said, Sh. (2017). Hacer política desde la escuela: narrativas biográficas de jóvenes en Argentina. Revista Latinoamericana de Ciencias Sociales, Niñez y Juventud, 15(2), 1085-1096. doi:10.11600/1692715x.1521920122016

Lahire, B. (2004). El hombre plural. Barcelona: Bellaterra.

Larrondo, M. (2017). Participación y escolarización de la política: reflexiones sobre lo político en la escuela. Universitas, XV(26), 109-134. Recuperado de: http://doi.org/10.17163/uni.n26.2017.04

Luci, F. (2012). El management como gramática: la producción de los dirigentes de empresas. Revista de Ciencias Sociales, 135-136, 171-183.

Martínez, M. E., Villa, A., \& Seoane, V. (Coords.) (2009). Jóvenes, elección escolar y distinción social. Buenos Aires: Prometeo.

Malinowski, B. (1987). «Introducción». Los argonautas del Pacífico Occidental. Barcelona: Planeta.

Mazzini, M. V. (2013). Palabras lindas, crudas realidades. Reflexiones sobre la enseñanza y el aprendizaje de la ideología empresarial. Buenos Aires: Ides-CAS.

Méndez, A. (2013). El colegio. Buenos Aires: Sudamericana.

Merleau-Ponty, M. (1945). Phénoménologie de la perception. París: Gallimard.

Núñez, P. (2013). La política en la escuela. Buenos Aires: La Crujía.

Núñez, P., \& Litichever L. (2015). Radiografías de la experiencia escolar. Buenos Aires: GEU.

Pasquali, P. (2014). Passer les frontières sociales, París: Fayard.

Poupeau, F. (2007). Dominación y movilizaciones. Córdoba: Ferreyra Editor.

Rockwell, E. (2009). La experiencia etnográfica. Buenos Aires: Paidós.

Tiramonti, G., \& Ziegler, S. (2008). La educación de las elites. Buenos Aires: Paidós.

Vázquez, M., Vommaro, P., Núñez, P., \& Blanco, R. (Eds.) (2017). Militancias juveniles en la 
Argentina democrática. Buenos Aires: Imago Mundi.

Vommaro, G., \& Morresi, S. D. (Orgs.) (2015). Hagamos equipo: la construcción de PRO y la renovación de la centroderecha argentina. Los Polvorines: UNGS.

Vommaro, P. (2015). Juventudes y politicas en la Argentina y en América Latina. Buenos Aires: GEU. Wilkis, A. (2013). Las sospechas del dinero. Buenos Aires: Paidós.

Ziegler, S., \& Gessaghi, V. (Comps.) (2012). Formación de las elites. Buenos Aires: Manantial, Flacso. 